TRANSACTIONS OF THE

AMERICAN MATHEMATICAL SOCIETY

Volume 356, Number 10, Pages 3951-3961

S 0002-9947(04)03436-

Article electronically published on February 4, 2004

\title{
WHEN DOES THE SUBADDITIVITY THEOREM FOR MULTIPLIER IDEALS HOLD?
}

\author{
SHUNSUKE TAKAGI AND KEI-ICHI WATANABE
}

\begin{abstract}
Demailly, Ein and Lazarsfeld proved the subadditivity theorem for multiplier ideals on nonsingular varieties, which states the multiplier ideal of the product of ideals is contained in the product of the individual multiplier ideals. We prove that, in the two-dimensional case, the subadditivity theorem holds on log terminal singularities. However, in the higher dimensional case, we have several counterexamples. We consider the subadditivity theorem for monomial ideals on toric rings and construct a counterexample on a threedimensional toric ring.
\end{abstract}

\section{INTRODUCTION}

Multiplier ideals were first introduced in the complex analytic context in the work of Demailly, Nadel, Siu and others, and they proved a Kodaira-type vanishing theorem involving these ideals. Multiplier ideals can be reformulated in a purely algebro-geometric setting in terms of resolution of singularities and discrepancy divisors, and nowadays this notion has become a fundamental tool in birational geometry.

Demailly, Ein and Lazarsfeld [DEL] proved the subadditivity theorem for multiplier ideals on nonsingular varieties, which states the multiplier ideal of the product of ideals is contained in the product of the individual multiplier ideals. This theorem itself is miraculous for commutative algebraists, and, moreover, it has several interesting applications to commutative algebra and algebraic geometry. For example, the problem concerning the growth of symbolic powers of ideals in regular local rings (see ELS]), Fujita's approximation theorem which asserts that most of the volume of a big divisor can be accounted for by the volume of an ample $\mathbb{Q}$-divisor on a modification (see $[\mathrm{Fu}]$ and $[\mathrm{La}]$ ), etc. However, their proof of the subadditivity theorem works only on nonsingular varieties over a field of characteristic zero, because their proof needs the Kawamata-Viehweg vanishing theorem and the fact that the diagonal embedding is a complete intersection.

Hence we investigate when the subadditivity theorem holds on singular varieties which have a resolution of singularities. The multiplier ideal associated to the unit ideal defines the locus of non-log-terminal points. Therefore, on non-log-terminal

Received by the editors January 2, 2003 and, in revised form, June 3, 2003.

2000 Mathematics Subject Classification. Primary 13B22; Secondary 14J17.

The authors thank MSRI for the support and hospitality during their stay in the fall of 2002 . The second author was partially supported by Grants-in-Aid in Scientific Researches, 13440015, 13874006; and his stay at MSRI was supported by the Bunri Fund, Nihon University. 
singularities, the subadditivity theorem fails. Conversely, in the two-dimensional case, using a characterization of integrally closed ideals in terms of anti-nef cycles, we show that the subadditivity theorem holds on log terminal singularities which are not necessarily essentially of finite type over a field of characteristic zero.

Theorem 2.2. Let $(A, \mathfrak{m})$ be a two-dimensional $\mathbb{Q}$-Gorenstein normal local ring. Then $A$ is log terminal if and only if the subadditivity theorem holds, that is, for any two ideals $\mathfrak{a}, \mathfrak{b} \subseteq A$,

$$
\mathcal{J}(\mathfrak{a} \mathfrak{b}) \subseteq \mathcal{J}(\mathfrak{a}) \mathcal{J}(\mathfrak{b})
$$

However, in the higher dimensional case, we have several counterexamples to Theorem 2.2 (See Example 3.1). Hence we investigate the subadditivity theorem for monomial ideals. The multiplier ideal associated to a monomial ideal is characterized by the Newton polygon (see $[\mathrm{HY}]$ and $[\mathrm{How}$ ) and it is easy to calculate this ideal. We expected that the subadditivity theorem for monomial ideals might hold on all toric rings. However, unfortunately, we found a counterexample on a three-dimensional toric ring (see Example 3.2).

\section{Multiplier ideals}

Notation. Throughout this paper, let $(A, \mathfrak{m})$ be an excellent normal $\mathbb{Q}$-Gorenstein local ring satisfying one of the following conditions:

- $(A, \mathfrak{m})$ is two-dimensional.

- $(A, \mathfrak{m})$ is essentially of finite type over a field of characteristic zero.

First we recall the definition of multiplier ideals. Refer to [La] for the general theory of multiplier ideals.

Definition 1.1. Let $\mathfrak{a}$ be an ideal in $A$. By [Hi], Li1] and [Li2], there exists a resolution of singularities $f: X \rightarrow \operatorname{Spec} A$ such that the ideal sheaf $\mathfrak{a} \mathcal{O}_{X}=\mathcal{O}_{X}(-F)$ is invertible and $\operatorname{Exc}(f) \cup \operatorname{Supp} F$ is a simple normal crossing divisor, where $\operatorname{Exc}(f)$ is the exceptional locus of $f$. Fix a rational number $c>0$. Then the multiplier idea 1 associated to $c$ and $\mathfrak{a}$ is defined to be

$$
\mathcal{J}\left(\mathfrak{a}^{c}\right)=\mathcal{J}\left(A, \mathfrak{a}^{c}\right)=H^{0}\left(X, \mathcal{O}_{X}\left(\left\lceil K_{X / \operatorname{Spec} A}-c F\right\rceil\right)\right) \subseteq A,
$$

where $K_{X / \operatorname{Spec} A}$ is the relative canonical divisor of $f$. In particular, $A$ is said to be a log terminal singularity if $\mathcal{J}(A)=A$.

Similarly, we can also define the multiplier ideal $\mathcal{J}\left(\mathfrak{a}^{c} \mathfrak{b}^{d}\right)$ associated to two ideals $\mathfrak{a}, \mathfrak{b}$ in $A$ and two rational numbers $c, d>0$ : Let $f: X \rightarrow$ Spec $A$ be a resolution of singularities such that $\mathfrak{a} \mathcal{O}_{X}=\mathcal{O}_{X}\left(-F_{a}\right)$ and $\mathfrak{b} \mathcal{O}_{X}=\mathcal{O}_{X}\left(-F_{b}\right)$ are invertible and $\operatorname{Exc}(f) \cup \operatorname{Supp} F_{a} \cup \operatorname{Supp} F_{b}$ is a simple normal crossing divisor. Then

$$
\mathcal{J}\left(\mathfrak{a}^{c} \mathfrak{b}^{d}\right)=H^{0}\left(X, \mathcal{O}_{X}\left(\left\lceil K_{X / \operatorname{Spec} A}-c F_{a}-d F_{b}\right\rceil\right)\right) \subseteq A .
$$

Remark 1.2. (1) Multiplier ideals are independent of the choice of a desingularization $f: X \rightarrow \operatorname{Spec} A$.

(2) Log terminal singularities are rational singularities.

\footnotetext{
${ }^{1}$ Lipman [Li3] calls this ideal the "adjoint ideal". However, Lazarsfeld [La] uses the term "adjoint ideal" in a different sense. To avoid confusion, we adopt the term "multiplier ideal" in this paper.
} 
The following basic properties of multiplier ideals immediately follow.

Proposition 1.3. Let $\mathfrak{a}$ and $\mathfrak{b}$ be ideals in $A$, and $c>0$ be a rational number.

(i) If $\mathfrak{a} \subseteq \mathfrak{b}$, then $\mathcal{J}\left(\mathfrak{a}^{c}\right) \subseteq \mathcal{J}\left(\mathfrak{b}^{c}\right)$.

(ii) $\mathcal{J}\left(\mathfrak{a}^{c}\right)$ is integrally closed. Moreover, $\mathcal{J}\left(\mathfrak{a}^{c}\right)=\mathcal{J}\left(\overline{\mathfrak{a}}^{c}\right)$, where we denote by $\overline{\mathfrak{a}}$ the integral closure of $\mathfrak{a}$.

(iii) Suppose that $A$ is a log terminal singularity. Then $\mathfrak{a} \subseteq \mathcal{J}(\mathfrak{a})$. Furthermore, if $\mathfrak{a}$ is an ideal of pure height one, then $\mathcal{J}(\mathfrak{a})=\mathfrak{a}$.

Proof. We will show only (iii). Let $f: X \rightarrow \operatorname{Spec} A$ be a resolution of singularities such that $\mathfrak{a} \mathcal{O}_{X}=\mathcal{O}_{X}(-Z)$ is invertible and $\operatorname{Exc}(f) \cup \operatorname{Supp} Z$ is a simple normal crossing divisor. Since $A$ is $\log$ terminal,

$$
\mathcal{J}(\mathfrak{a})=H^{0}\left(X, \mathcal{O}_{X}\left(\left\lceil K_{X / \operatorname{Spec} A}-Z\right\rceil\right)\right) \supseteq H^{0}\left(X, \mathcal{O}_{X}(-Z)\right)=\overline{\mathfrak{a}} .
$$

Since $\operatorname{codim}_{A}(\operatorname{Supp}(\mathcal{J}(\mathfrak{a}) / \overline{\mathfrak{a}})) \geq 2$, we have $\mathfrak{a}=\overline{\mathfrak{a}}=\mathcal{J}(\mathfrak{a})$ when $\mathfrak{a}$ is divisorial.

Demailly, Ein and Lazarsfeld proved the following theorem, which is called the subadditivity theorem.

Theorem 1.4 ([DEL]). Let $A$ be a regular local ring essentially of finite type over a field of characteristic zero, and let $\mathfrak{a}$ and $\mathfrak{b}$ be any two ideals in A. Fix any rational numbers $c, d>0$. Then

$$
\mathcal{J}\left(\mathfrak{a}^{c} \mathfrak{b}^{d}\right) \subseteq \mathcal{J}\left(\mathfrak{a}^{c}\right) \mathcal{J}\left(\mathfrak{b}^{d}\right) .
$$

Remark 1.5. Demailly, Ein and Lazarsfeld use the Kawamata-Viehweg vanishing theorem, hence the condition that $A$ is essentially of finite type over a field of characteristic zero is necessary for their proof.

In this paper, we say that the subadditivity theorem holds if $\mathcal{J}(\mathfrak{a} \mathfrak{b}) \subseteq \mathcal{J}(\mathfrak{a}) \mathcal{J}(\mathfrak{b})$ for any ideals $\mathfrak{a}, \mathfrak{b} \subseteq A$, and the strong subadditivity theorem holds if $\mathcal{J}\left(\mathfrak{a}^{c} \mathfrak{b}^{d}\right) \subseteq$ $\mathcal{J}\left(\mathfrak{a}^{c}\right) \mathcal{J}\left(\mathfrak{b}^{d}\right)$ for any $\mathfrak{a}, \mathfrak{b}$ and any rational numbers $c, d>0$.

\section{TwO-DimensionAL CASE}

In this section, we investigate when the subadditivity theorem holds in the twodimensional case. The following characterization of integrally closed ideals is quite useful.

Theorem 2.1 (Li1], Gi]). Let $(A, \mathfrak{m})$ be a two-dimensional rational singularity and fix a resolution of singularities $f: X \rightarrow \operatorname{Spec} A$ with $E$ the exceptional locus on $X$. Let $E_{1}, \ldots, E_{r}$ be all the irreducible components of $E$. Then there is a oneto-one correspondence between the set of integrally closed ideals $I$ in A such that $I \mathcal{O}_{X}$ is invertible and the set of effective $f$-anti-nef cycles $Z$ on $X$ (i.e. $Z \geq 0$ and $Z \cdot E_{i} \leq 0$ for all $\left.1 \leq i \leq r\right)$. The correspondence is given by $I_{\mathcal{O}_{X}}=\mathcal{O}_{X}(-Z)$ and $I=H^{0}\left(X, \mathcal{O}_{X}(-Z)\right)$.

Using the above theorem, we obtain necessary and sufficient conditions for the subadditivity theorem to hold.

Theorem 2.2. Let $(A, \mathfrak{m})$ be a two-dimensional $\mathbb{Q}$-Gorenstein normal local ring. Then $A$ is a log terminal singularity if and only if the subadditivity theorem holds, that is, for any two ideals $\mathfrak{a}, \mathfrak{b} \subseteq A$,

$$
\mathcal{J}(\mathfrak{a} \mathfrak{b}) \subseteq \mathcal{J}(\mathfrak{a}) \mathcal{J}(\mathfrak{b}) .
$$


Proof. If the subadditivity theorem holds, then $\mathcal{J}(A) \subseteq \mathcal{J}(A)^{2}$. Thus $\mathcal{J}(A)=A$, namely $A$ is $\log$ terminal. Hence we will show the converse implication, that is, we will prove that for any two ideals $\mathfrak{a}, \mathfrak{b} \subseteq A, \mathcal{J}(\mathfrak{a} \mathfrak{b}) \subseteq \mathcal{J}(\mathfrak{a}) \mathcal{J}(\mathfrak{b})$ when $A$ is a $\log$ terminal singularity. By Proposition 1.3 (ii), we may assume that $\mathfrak{a}$ and $\mathfrak{b}$ are integrally closed. Let $f: X \rightarrow \operatorname{Spec} A$ be a resolution of singularities such that $\mathfrak{a} \mathcal{O}_{X}=\mathcal{O}_{X}\left(-F_{a}\right)$ and $\mathfrak{b} \mathcal{O}_{X}=\mathcal{O}_{X}\left(-F_{b}\right)$ are invertible and $\operatorname{Exc}(f) \cup \operatorname{Supp} F_{a} \cup$ Supp $F_{b}$ is a simple normal crossing divisor. By Theorem 2.1, $F_{a}$ and $F_{b}$ are $f$-antinef cycles on $X$, which are not necessarily supported on the exceptional locus of $f$. By the definition of multiplier ideals, denoting by $K$ the relative canonical divisor $K_{X / \operatorname{Spec} A}$ of $f$, we have

$$
\begin{aligned}
\mathcal{J}(\mathfrak{a}) \mathcal{J}(\mathfrak{b}) & =H^{0}\left(X, \mathcal{O}_{X}\left(\lceil K\rceil-F_{a}\right)\right) \cdot H^{0}\left(X, \mathcal{O}_{X}\left(\lceil K\rceil-F_{b}\right)\right), \\
\mathcal{J}(\mathfrak{a} \mathfrak{b}) & =H^{0}\left(X, \mathcal{O}_{X}\left(\lceil K\rceil-F_{a}-F_{b}\right)\right) .
\end{aligned}
$$

Here, for every cycle $Z$ on $X$, we denote by $\operatorname{an}_{f}(Z)$ the $f$-anti-nef closure of $Z$, namely the minimal $f$-anti-nef cycle among all cycles on $X$ which are bigger than or equal to $Z$. Note that $\operatorname{an}_{f}(Z)$ is uniquely determined by $Z$ (cf. $\operatorname{Ar}$ ). Since $A$ is a rational singularity, the product of integrally closed ideals of $A$ is also integrally closed [Li1]. Hence $\mathcal{J}(\mathfrak{a}) \mathcal{J}(\mathfrak{b})$ and $\mathcal{J}(\mathfrak{a} \mathfrak{b})$ are integrally closed, and by Theorem 2.1 again, $\mathcal{J}(\mathfrak{a}) \mathcal{J}(\mathfrak{b})$ and $\mathcal{J}(\mathfrak{a} \mathfrak{b})$ correspond to the cycles $\operatorname{an}_{f}\left(F_{a}-\lceil K\rceil\right)+\operatorname{an}_{f}\left(F_{b}-\lceil K\rceil\right)$ and $\operatorname{an}_{f}\left(F_{a}+F_{b}-\lceil K\rceil\right)$, respectively. Therefore, it suffices to show that

$$
\operatorname{an}_{f}\left(F_{a}-\lceil K\rceil\right)+\operatorname{an}_{f}\left(F_{b}-\lceil K\rceil\right) \leq \operatorname{an}_{f}\left(F_{a}+F_{b}-\lceil K\rceil\right) .
$$

In order to prove this, we prepare some notation. We can assume that the residue field $A / \mathfrak{m}$ is algebraically closed. Then the morphism $f$ can be factorized as

$$
X:=X_{n} \stackrel{f_{n}}{\longrightarrow} X_{n-1} \stackrel{f_{n-1}}{\longrightarrow} \cdots \stackrel{f_{1}}{\longrightarrow} X_{0} \stackrel{f_{0}}{\longrightarrow} \operatorname{Spec} A,
$$

where $f_{i}: X_{i} \rightarrow X_{i-1}$ is a contraction of a $(-1)$-curve $E_{i}$ on $X_{i}$ for every $i=1, \ldots, n$ and $f_{0}: X_{0} \rightarrow \operatorname{Spec} A$ is the minimal resolution of $\operatorname{Spec} A$. We denote by $\pi_{i}: X \rightarrow$ $X_{i}$ the composite of $f_{i+1}, \ldots, f_{n}$ for $i=0,1, \ldots, n-1$ and by $\pi_{i, j}: X_{i} \rightarrow X_{j}$ the composite of $f_{j+1}, \ldots, f_{i}$ for $i>j$. Then the relation $\triangleright$ on $f$-exceptional divisors $E_{1}, \ldots, E_{n}$ is defined as follows: $E_{i} \triangleright E_{j}$ if and only if the intersection number $\pi_{i}{ }^{*} E_{i} \cdot \pi_{j_{*}}{ }^{-1} E_{j}$ is positive, where $\pi_{j_{*}}^{-1} E_{j}$ is the strict transform of $E_{j}$ on $X$. Since the relation $\triangleright$ is not a order relation, we denote by $>$ the order relation generated by $\triangleright$. by

Let $\mathbf{P}$ be the proximity matrix of $f$, that is, the matrix $\mathbf{P}:=\left(p_{i j}\right)_{1 \leq i, j \leq n}$ given

$$
p_{i j}=\left\{\begin{array}{cl}
1 & \text { if } i=j, \\
-1 & \text { if } E_{j} \triangleright E_{i}, \\
0 & \text { otherwise }
\end{array}\right.
$$

(see [DV] and [Li4]).

Claim 1. Let $Z$ be a cycle on $X$ and we write

$$
Z=\pi_{0}{ }^{*} \pi_{0 *} Z+\sum_{i=1}^{n} d_{i} \pi_{i}^{*} E_{i}
$$

Then $Z$ is $f$-anti-nef if and only if $\pi_{0 *} Z$ is an $f_{0}$-anti-nef cycle on $X_{0}$ and every component of $\mathbf{P d}$ is a nonnegative integer, where $\mathbf{d}={ }^{t}\left(d_{1}, \ldots, d_{n}\right)$. In particular, if $Z$ is $f$-anti-nef, then $d_{i} \geq 0$ for every $i$. 
Proof of Claim 1. Since $Z=\pi_{j-1}{ }^{*} \pi_{j-1_{*}} Z+\sum_{i=j}^{n} d_{i} \pi_{i}{ }^{*} E_{i}$ for every $j=1,2, \ldots, n$,

$$
Z \cdot \pi_{j_{*}}^{-1} E_{j}=\sum_{i=j}^{n} d_{i} E_{i} \cdot \pi_{i, j_{*}}^{-1} E_{j}=-\mathbf{e}_{j} \mathbf{P d},
$$

where $\mathbf{e}_{j}$ is the $n$-tuple (row) vector such that the $j$-th component is one and the other components are zero. On the other hand, for each $f_{0}$-exceptional curve $F$, we have $Z \cdot \pi_{0}{ }^{*} F=\pi_{0 *} Z \cdot F$. Therefore, $Z$ is $f$-anti-nef if and only if $\mathbf{e}_{j} \mathbf{P d}$ is nonnegative for all $j=1, \ldots, n$ and $\pi_{0 *} Z$ is $f_{0}$-anti-nef.

Fix any effective $f$-anti-nef cycle $Z$ on $X$ and write $Z=\pi_{0}{ }^{*} \pi_{0 *} Z+\sum_{i=1}^{n} d_{i} \pi_{i}{ }^{*} E_{i}$. Then we investigate a process for computation of the anti-nef closure of $Z-\lceil K\rceil$. Let $\Lambda$ be a subset of $\{1,2, \ldots, n\}$ such that $\lceil K\rceil=\sum_{i \in \Lambda} \pi_{i}{ }^{*} E_{i}$. Then define $Z^{(k)}:=\pi_{0}{ }^{*} \pi_{0 *} Z+\sum_{i=1}^{n} d_{i}^{(k)} \pi_{i}{ }^{*} E_{i}$ inductively as follows: Let

$$
d_{i}^{(0)}= \begin{cases}d_{i}-1 & \text { if } i \in \Lambda \text { and } d_{i}>0 \\ d_{i} & \text { otherwise }\end{cases}
$$

for all $i=1, \ldots, n$. If $Z^{(1)}, \ldots, Z^{(k-1)}$ are defined and if $\mathbf{e}_{j} \mathbf{P} \mathbf{d}^{(k-1)}$ is negative for some $1 \leq j \leq n$ where $\mathbf{d}^{(k-1)}={ }^{t}\left(d_{1}^{(k-1)}, \ldots, d_{n}^{(k-1)}\right)$, then choose one of such $j$ (we denote this by the same letter $j$ ) and set

$$
d_{i}^{(k)}= \begin{cases}d_{j}^{(k-1)}+1 & \text { if } i=j \\ d_{i}^{(k-1)}-1 & \text { if } E_{i} \triangleright E_{j} \text { and } d_{i}^{(k-1)}>0 \\ d_{i}^{(k-1)} & \text { otherwise. }\end{cases}
$$

Claim 2. This process stops after finitely many steps. When the process stops at $Z^{\left(k_{0}\right)}$, the cycle $Z^{\left(k_{0}\right)}$ is the $f$-anti-nef closure of $Z-\lceil K\rceil$.

Proof of Claim 2. This is similar to the computation of the fundamental cycle (see $(\mathrm{Ar})$. So we give only one remark here. Some readers may think that for the minimality of the anti-nef closure of $Z-\lceil K\rceil, d_{i}^{(0)}$ should be defined as follows:

$$
d_{i}^{(0)}= \begin{cases}d_{i}-1 & \text { if } i \in \Lambda \text { and } d_{i}>0, \\ d_{i}-1 & \text { if } d_{i}>0 \text { and if } d_{j}=0 \text { for some } j \in \Lambda \text { such that } E_{i} \triangleright E_{j}, \\ d_{i} & \text { otherwise. }\end{cases}
$$

However, this definition coincides with the one above. In fact, since $Z$ is an $f$ anti-nef cycle, once $d_{j}=0$, we have $d_{i}=0$ for all $i$ such that $E_{i} \triangleright E_{j}$. Therefore, when $d_{i}>0$, there exists no such $E_{i} \triangleright E_{j}$ as $d_{j}=0$.

In this paper, we call such a sequence of cycles as $\left\{Z^{(0)}, \ldots, Z^{\left(k_{0}\right)}\right\}$ a computation sequence for $Z-\lceil K\rceil$ (there may be several computation sequences).

Claim 3. $d_{i}-1 \leq d_{i}^{(k)} \leq d_{i}$ for every $i=1, \ldots, n$ and $k=0,1, \ldots, k_{0}$. Therefore, $\mathbf{e}_{j} \mathbf{P} \mathbf{d}^{(k)}<0$ if and only if $\mathbf{e}_{j} \mathbf{P d}=0, d_{j}^{(k)}=d_{j}-1$ and $d_{i}^{(k)}=d_{i}$ for all $i$ such that $E_{i} \triangleright E_{j}$.

Proof of Claim 3. Since $Z$ is $f$-anti-nef, $\operatorname{an}_{f}(Z-\lceil K\rceil) \leq Z$ and $d_{i}^{(k)} \leq d_{i}$. We will show that $d_{i}-1 \leq d_{i}^{(k)}$ by induction on $k$. When $k=0$, the assertion is trivial. Hence we may assume that $k \geq 1$. By the induction hypothesis, $d_{i}-1 \leq$ $d_{i}^{(k-1)} \leq d_{i}$. If $d_{i}^{(k-1)}=d_{i}-1$, then for every $E_{j} \triangleleft E_{i}, \mathbf{e}_{j} \mathbf{P d}^{(k-1)} \geq 0$. Therefore, $d_{i}^{(k)} \geq d_{i}-1$. 
Now using this process, we will prove the inequality (2.1). Write

$$
\begin{aligned}
& F_{a}=\pi_{0}{ }^{*} \pi_{0 *} F_{a}+\sum_{i=1}^{n} a_{i} \pi_{i}{ }^{*} E_{i}, \\
& F_{b}=\pi_{0}{ }^{*} \pi_{0 *} F_{b}+\sum_{i=1}^{n} b_{i} \pi_{i}{ }^{*} E_{i},
\end{aligned}
$$

and denote $\mathbf{a}={ }^{t}\left(a_{1}, \ldots, a_{n}\right)$ and $\mathbf{b}={ }^{t}\left(b_{1}, \ldots, b_{n}\right)$.

Let $F_{c}:=F_{a}+F_{b}$ and apply the above process to $F_{c}$. Then we get a computation sequence $\left\{F_{c}^{(0)}, \ldots, F_{c}^{\left(k_{c}\right)}\right\}$ (we denote $F_{c}^{(k)}:=\pi_{0}{ }^{*} \pi_{0 *}\left(F_{a}+F_{b}\right)+\sum_{i=1}^{n} c_{i}^{(k)} \pi_{i}{ }^{*} E_{i}$ for $\left.0 \leq k \leq k_{c}\right)$ for $F_{a}+F_{b}-\lceil K\rceil$. We denote $c^{(k)}={ }^{t}\left(c_{1}^{(k)}, \ldots, c_{n}^{(k)}\right)$ for all $0 \leq k \leq k_{c}$. We define $\mathbf{a}^{(0)}$ and $\mathbf{b}^{(0)}$ as in (2.2).

By definition and Claim 3, $\mathbf{e}_{j} \mathbf{P} \mathbf{c}^{(0)}<0$ if and only if $\mathbf{e}_{j} \mathbf{P}(\mathbf{a}+\mathbf{b})=0, j \in \Lambda$, $a_{j}+b_{j}>0$ and $a_{i}+b_{i}=0$ for every $i \in \Lambda$ such that $E_{i} \triangleright E_{j}$. Therefore, the condition $\mathbf{e}_{j} \mathbf{P} \mathbf{c}^{(0)}<0$ implies $\mathbf{e}_{j} \mathbf{P a}{ }^{(0)}<0$ and $\mathbf{e}_{j} \mathbf{P} \mathbf{b}^{(0)}<0$, unless $a_{j}$ or $b_{j}$ is zero. If $c_{j}^{(1)}=c_{j}^{(0)}+1$, then we define $F_{a}^{(1)}:=\pi_{0}{ }^{*} \pi_{0 *} F_{a}+\sum_{i=1}^{n} a_{i}^{(1)} \pi_{i}{ }^{*} E_{i}$ (resp. $F_{b}^{(1)}:=\pi_{0}{ }^{*} \pi_{0 *} F_{b}+\sum_{i=1}^{n} b_{i}^{(1)} \pi_{i}{ }^{*} E_{i}$ ) as follows (cf. (2.3)): If $a_{j}=0$ (resp. $b_{j}=0$ ), then set $F_{a}^{(1)}=F_{a}^{(0)}\left(\right.$ resp. $\left.F_{b}^{(1)}=F_{b}^{(0)}\right)$. If $a_{j}>0\left(\right.$ resp. $\left.b_{j}>0\right)$, then

$$
\begin{aligned}
& a_{i}^{(1)}= \begin{cases}a_{i}^{(0)}+1 & \text { if } i=j, \\
a_{i}^{(0)}-1 & \text { if } E_{i} \triangleright E_{j} \text { and } a_{i}^{(0)}>0, \\
a_{i}^{(0)} & \text { otherwise }\end{cases} \\
& \left(\text { resp. } b_{i}^{(1)}=\left\{\begin{array}{ll}
b_{i}^{(0)}+1 & \text { if } i=j, \\
b_{i}^{(0)}-1 & \text { if } E_{i} \triangleright E_{j} \text { and } b_{i}^{(0)}>0, \\
b_{i}^{(0)} & \text { otherwise }
\end{array}\right)\right. \text {. }
\end{aligned}
$$

We can inductively define a cycle $F_{a}^{(k)}\left(\right.$ resp. $\left.F_{b}^{(k)}\right)$ for every $k=1, \ldots, k_{c}$ as above. When $a_{j}$ and $b_{j}$ are nonzero integers, $\mathbf{e}_{j} \mathbf{P} \mathbf{c}^{(k)}<0$ if and only if $\mathbf{e}_{j} \mathbf{P a}^{(k)}<0$ and $\mathbf{e}_{j} \mathbf{P} \mathbf{b}^{(k)}<0$ for each $k=0,1, \ldots, k_{c}$. Hence, $F_{a}^{\left(k_{c}\right)}$ (resp. $F_{b}^{\left(k_{c}\right)}$ ) is not necessarily $f$-anti-nef, but $\left\{F_{a}^{(1)}, \ldots, F_{a}^{\left(k_{c}\right)}\right\}$ (resp. $\left.\left\{F_{b}^{(1)}, \ldots, F_{b}^{\left(k_{c}\right)}\right\}\right)$ can be extended to a computation sequence $\left\{F_{a}^{(1)}, \ldots, F_{a}^{\left(k_{c}\right)}, \ldots, F_{a}^{\left(k_{a}\right)}\right\}$ (resp. $\left.\left\{F_{b}^{(1)}, \ldots, F_{b}^{\left(k_{c}\right)}, \ldots, F_{b}^{\left(k_{b}\right)}\right\}\right)$ for $F_{a}-\lceil K\rceil$ (resp. $F_{b}-\lceil K\rceil$ ). Moreover, if $a_{i}$ and $b_{i}$ are nonzero, then by Claim 3 , the triple $\left(a_{i}^{\left(k_{c}\right)}, b_{i}^{\left(k_{c}\right)}, c_{i}^{\left(k_{c}\right)}\right)$ coincides with one of the following:

$$
\left(a_{i}, b_{i}, a_{i}+b_{i}\right),\left(a_{i}-1, b_{i}-1, a_{i}+b_{i}-1\right) .
$$

Then we will show that $a_{i}^{\left(k_{a}\right)}+b_{i}^{\left(k_{b}\right)} \leq c_{i}^{\left(k_{c}\right)}$.

If $\left(a_{i}^{\left(k_{c}\right)}, b_{i}^{\left(k_{c}\right)}, c_{i}^{\left(k_{c}\right)}\right)=\left(a_{i}, b_{i}, a_{i}+b_{i}\right)$, then, by Claim 3, we have $a_{i}^{\left(k_{a}\right)}+b_{i}^{\left(k_{b}\right)} \leq$ $a_{i}+b_{i}=c_{i}^{\left(k_{c}\right)}$

If $b_{i}=0$, then $\left(a_{i}^{\left(k_{c}\right)}, b_{i}^{\left(k_{c}\right)}, c_{i}^{\left(k_{c}\right)}\right)=\left(a_{i}-1,0, a_{i}-1\right)$. Then, we have $b_{j}=0$ for all $j$ such that $E_{j}>E_{i}$, because $F_{b}$ is $f$-anti-nef. Hence $b_{i}^{(k)}=b_{i}=0$ for all $k_{c} \leq k \leq k_{b}$ and $a_{i}^{(k)}=c_{i}^{\left(k_{c}\right)}=a_{i}-1$ for every $k_{c} \leq k \leq k_{a}$, in particular, $a_{i}^{\left(k_{a}\right)}+b_{i}^{\left(k_{b}\right)}=a_{i}-1=c_{i}^{\left(k_{c}\right)}$. The case where $a_{i}=0$ is similar.

Thus we suppose that $\left(a_{i}^{\left(k_{c}\right)}, b_{i}^{\left(k_{c}\right)}, c_{i}^{\left(k_{c}\right)}\right)=\left(a_{i}-1, b_{i}-1, a_{i}+b_{i}-1\right)$. Then it suffices to prove that there exist no such $p, q \geq k_{c}$ as $a_{i}^{(p)}=a_{i}$ and $b_{i}^{(q)}=b_{i}$. 
Assume to the contrary that $a_{i}^{(p)}=a_{i}$ and $b_{i}^{(q)}=b_{i}$ for some $p, q \geq k_{c}$. Then among the index $j$ 's for which $\left(a_{j}^{\left(k_{c}\right)}, b_{j}^{\left(k_{c}\right)}, c_{j}^{\left(k_{c}\right)}\right)=\left(a_{j}-1, b_{j}-1, a_{j}+b_{j}-1\right), a_{j}^{(p)}=a_{j}$ and $b_{j}^{(q)}=b_{j}$ for some $p, q \geq k_{c}$, take $j$ such that $E_{j}$ is maximal with respect to the relation $>$.

If $\mathbf{e}_{j} \mathbf{P a} \mathbf{a}^{\left(k_{c}\right)}<0$ and $\mathbf{e}_{j} \mathbf{P} \mathbf{b}^{\left(k_{c}\right)}<0$, then $\mathbf{e}_{j} \mathbf{P} \mathbf{c}^{\left(k_{c}\right)}<0$, that is, $F_{c}^{\left(k_{c}\right)}$ is not $f$ anti-nef. Therefore if $\mathbf{e}_{j} \mathbf{P a}{ }^{\left(k_{c}\right)}<0$, then $\mathbf{e}_{j} \mathbf{P b}^{\left(k_{c}\right)} \geq 0$, which implies by Claim 3 that $\mathbf{e}_{j} \mathbf{P b}>0$ or there exists $E_{h} \triangleright E_{j}$ such that $a_{h}=0$. Notice that, in the latter case, $b_{h}^{(k)}=b_{h}-1$ for every $k_{b} \geq k \geq k_{c}$. Thus, in both cases, $\mathbf{e}_{j} \mathbf{P b}^{(k)} \geq 0$ for all $k_{b} \geq k \geq k_{c}$, namely $b_{j}^{(k)}=b_{j}-1$ for every $k_{b} \geq k \geq k_{c}$.

Hence, in order that $a_{j}^{(p)}=a_{j}$ and $b_{j}^{(q)}=b_{j}$ for some $p, q \geq k_{c}, \mathbf{e}_{j} \mathbf{P a}^{\left(k_{c}\right)} \geq 0$, $\mathbf{e}_{j} \mathbf{P b}^{\left(k_{c}\right)} \geq 0$ and $\mathbf{e}_{j} \mathbf{P a}=\mathbf{e}_{j} \mathbf{P b}=0$. If there exists some $E_{h} \triangleright E_{j}$ such that $a_{h}=0$ or $b_{h}=0$, then it does not occur that $a_{j}^{(p)}=a_{j}$ and $b_{j}^{(q)}=b_{j}$ for some $p, q \geq k_{c}$.

Therefore, there exists $E_{h} \triangleright E_{j}$ such that

$$
\left(a_{h}^{\left(k_{c}\right)}, b_{h}^{\left(k_{c}\right)}, c_{h}^{\left(k_{c}\right)}\right)=\left(a_{h}-1, b_{h}-1, a_{h}+b_{h}-1\right),
$$

and in order that $a_{j}^{(p)}=a_{j}$ and $b_{j}^{(q)}=b_{j}$ for $p, q \geq k_{c}$, it is necessary that $a_{h}^{(s)}=a_{h}$ and $b_{h}^{(t)}=b_{h}$ for some $p>s>k_{c}$ and $q>t>k_{c}$. However, this contradicts the maximality of the index $j$. Thus we have $a_{i}^{\left(k_{a}\right)}+b_{i}^{\left(k_{b}\right)} \leq a_{i}+b_{i}-1=c_{i}^{\left(k_{c}\right)}$.

Remark 2.3. (1) If $A$ is a two-dimensional Gorenstein log terminal singularity, then the relative canonical divisor $K$ is an integral divisor. In this case, the anti-nef closure of $Z-K$ for an $f$-anti-nef cycle $Z$ can simply be described as follows:

$$
\operatorname{an}_{f}(Z-K)=Z-K+\sum_{\pi_{i *} Z \cdot E_{i}=0} \pi_{i}^{*} E_{i} .
$$

By this formula, one can see that there are many cases in which the equality in the subadditivity theorem fails (see Example 2.4 (1)). On the other hand, in the non-Gorenstein case, $\operatorname{an}_{f}(Z-\lceil K\rceil)$ is more complicated and an analog of the above formula

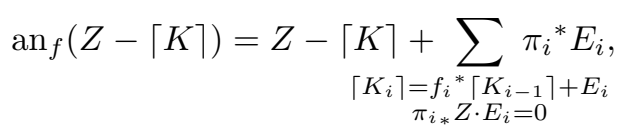

where $K_{i}$ is the relative canonical divisor of $f_{i}$, does not hold (see Example2.4 (2)).

(2) Multiplier ideals can also be defined for divisors (refer to [La] for details), and Demailly, Ein and Lazarsfeld [DEL] also proved the subadditivity theorem for divisors under the assumption that $A$ is regular. Some readers may expect that the subadditivity theorem for divisors also holds if $A$ is a two-dimensional $\log$ terminal singularity, but this is not true. For example, let $A=\mathbb{C}[[X, Y, Z]] /\left(X Y-Z^{2}\right)$, $\mathcal{O}_{\operatorname{Spec} A}\left(-D_{1}\right)=(x, z)$ and $\mathcal{O}_{\operatorname{Spec} A}\left(-D_{2}\right)=(y, z)$, where $x, y, z$ are the images of $X, Y, Z$ in $A$, respectively. Then $\mathcal{J}\left(D_{1}\right)=(x, z)$ and $\mathcal{J}\left(D_{2}\right)=(y, z)$, but $\mathcal{J}\left(D_{1}+D_{2}\right)=(z)$. Hence $\mathcal{J}\left(D_{1}+D_{2}\right) \nsubseteq \mathcal{J}\left(D_{1}\right) \mathcal{J}\left(D_{2}\right)$.

Example 2.4. (1) ( $A_{1}$-singularity) Let $A=\mathbb{C}[[X, Y, Z]] /\left(X Y-Z^{2}\right)$ and $\mathfrak{a}=$ $\left(x^{2}, y, z\right)$, where $x, y, z$ are the images of $X, Y, Z$ in $A$, respectively. Then

$$
\mathcal{J}(\mathfrak{a})=(x, y, z), \quad \mathcal{J}\left(\mathfrak{a}^{2}\right)=\left(x^{3}, y^{2}, z^{2}, x y, y z, z x\right) .
$$


Therefore, $\mathcal{J}\left(\mathfrak{a}^{2}\right) \subsetneq \mathcal{J}(\mathfrak{a})^{2}$. Indeed, let $f_{0}: X_{0} \rightarrow$ Spec $A$ be the minimal resolution with the irreducible exceptional curve $F$ and $f_{1}: X \rightarrow X_{0}$ the blowing-up of $X_{0}$ at a point on $F$. Let $f: X \rightarrow \operatorname{Spec} A$ be the composite morphism of $f_{0}$ and $f_{1}$. Then the relative canonical divisor $K$ of $f$ is $E_{1}$ and the ideal $\mathfrak{a}$ corresponds to the $f$-anti-nef cycle $F_{a}=2 E_{1}+F^{\prime}$, where $E_{1}$ is the unique $f_{1}$-exceptional curve and $F^{\prime}$ is the strict transform of $F$. Hence we have $\operatorname{an}_{f}\left(F_{a}-K\right)=E_{1}+F^{\prime}$ and $\operatorname{an}_{f}\left(2 F_{a}-K\right)=3 E_{1}+2 F^{\prime}$. Thus $2 \operatorname{an}_{f}\left(F_{a}-K\right) \lesseqgtr \operatorname{an}_{f}\left(2 F_{a}-K\right)$.

(2) Let $A=\mathbb{C}\left[X^{5}, X Y^{3}, X^{2} Y, Y^{5}\right] \subset \mathbb{C}[X, Y]$. We denote by $F_{1}$ and $F_{2}$ the exceptional curves of the minimal resolution $f_{0}: X_{0} \rightarrow \operatorname{Spec} A$ such that $F_{1}^{2}=-2$ and $F_{2}^{2}=-3$. Let $f_{1}: X_{1} \rightarrow X_{0}$ be the blowing-up at the intersection of $F_{1}$ and $F_{2}$ with the $f_{1}$-exceptional curve $E_{1}$, and let $f_{2}: X \rightarrow X_{1}$ be the blowing-up at the intersection of $E_{1}$ and the strict transform $F_{2}{ }^{\prime}$ of $F_{2}$ with the $f_{2}$-exceptional curve $E_{2}$. Let $f: X \rightarrow \operatorname{Spec} A$ be the composite morphism of $f_{0}, f_{1}$ and $f_{2}$. Then the graph of $f$-exceptional curves is the following:

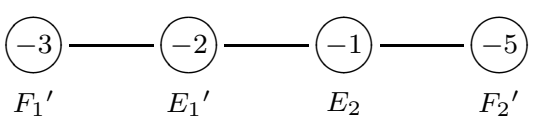

Fix an $f$-anti-nef cycle $Z=F_{1}{ }^{\prime}+3 E_{1}{ }^{\prime}+5 E_{2}+F_{2}{ }^{\prime}$ on $X$. Since the relative canonical divisor $K$ of $f$ is $-\frac{1}{5}{F_{1}}^{\prime}+\frac{2}{5} E_{1}{ }^{\prime}+E_{2}-\frac{2}{5} F_{2}{ }^{\prime}$, we have

$$
\begin{aligned}
& \operatorname{an}_{f}(Z-\lceil K\rceil)= \operatorname{an}_{f}\left(F_{1}{ }^{\prime}+2 E_{1}{ }^{\prime}+4 E_{2}+F_{2}{ }^{\prime}\right)=F_{1}{ }^{\prime}+3 E_{1}{ }^{\prime}+4 E_{2}+F_{2} \\
&=Z-\lceil K\rceil+E_{1}{ }^{\prime} \\
& \neq Z-\lceil K\rceil+\sum_{\substack{\left\lceil K_{i}\right\rceil=f_{i}{ }^{*}\left\lceil K_{i-1}\right\rceil+E_{i} \\
\pi_{i *} Z \cdot E_{i}=0}} \pi_{i}{ }^{*} E_{i} .
\end{aligned}
$$

On the other hand, in order that the strong subadditivity theorem holds, nonsingularness is necessary.

Proposition 2.5. Let $(A, \mathfrak{m})$ be a two-dimensional $\mathbb{Q}$-Gorenstein normal local ring such that the residue field $A / \mathfrak{m}$ is algebraically closed. If the strong subadditivity theorem holds, that is,

$$
\mathcal{J}\left(\mathfrak{a}^{c} \mathfrak{b}^{d}\right) \subseteq \mathcal{J}\left(\mathfrak{a}^{c}\right) \mathcal{J}\left(\mathfrak{b}^{d}\right)
$$

for any ideals $\mathfrak{a}, \mathfrak{b} \subseteq A$ and any rational numbers $c, d>0$, then $A$ is regular. In particular, when $A$ is essentially of finite type over a field of characteristic zero, $A$ is regular if and only if the strong subadditivity theorem holds.

Proof. Assume that $A$ is not regular. Let $f: X \rightarrow \operatorname{Spec} A$ be the minimal resolution, and then the exceptional locus $\operatorname{Exc}(f)$ of $f$ is not trivial. In order that the strong subadditivity theorem hold, by Theorem 2.2, it is necessary that $A$ is a log terminal singularity.

(1) The case where $\operatorname{Exc}(f)$ is irreducible.

Let $E$ be the unique irreducible $f$-exceptional curve. Then $E^{2}=-k$ for some integer $k \geq 2$. Let $g: Y \rightarrow X$ be the blowing-up at a point on the curve $E$ and $h: Y \rightarrow \operatorname{Spec} A$ the composite morphism of $f$ and $g$. We denote by $E_{1}$ the exceptional divisor of $g$ and by $E_{2}$ the strict transform of $E$. Then $E_{1}{ }^{2}=-1, E_{2}{ }^{2}=-k-1$ and the relative canonical divisor $K$ of $h$ is equal to $\frac{2}{k} E_{1}-\frac{k-2}{k} E_{2}$. Fix an $h$-anti-nef cycle $Z:=2(k+1) E_{1}+2 E_{2}$ 
on $Y$. Here, for every cycle $F$ on $Y$, we denote by $\operatorname{an}_{h}(F)$ the $h$-anti-nef closure of $F$ as in the proof of Theorem [2.2. Then

$$
\begin{aligned}
& \operatorname{an}_{h}\left(\left\lfloor\frac{1}{k+1} Z-K\right\rfloor\right)=\operatorname{an}_{h}\left(E_{1}\right)=E_{1}+E_{2}, \\
& \operatorname{an}_{h}\left(\left\lfloor\frac{2}{k+1} Z-K\right\rfloor\right)=\operatorname{an}_{h}\left(3 E_{1}+E_{2}\right)=3 E_{1}+E_{2} .
\end{aligned}
$$

Hence $\mathcal{J}\left(I^{\frac{2}{k+1}}\right) \not \mathcal{J}\left(I^{\frac{1}{k+1}}\right)^{2}$, where $I=H^{0}\left(Y, \mathcal{O}_{Y}(-Z)\right) \subset A$. This implies that the strong subadditivity theorem does not hold on $A$.

(2) The case where $\operatorname{Exc}(f)$ is reducible.

Let $Z_{f}$ be the fundamental cycle of $f$. Since $Z_{f}$ is reducible, we can take an $f$-anti-nef cycle $Z$ such that $Z_{f} \leq Z<n Z_{f}$ and $\left\lfloor\frac{1}{n} Z\right\rfloor \neq 0$ for some integer $n \geq 2$. We denote by $K_{0}$ the relative canonical divisor of $f$. Since $A$ is $\log$ terminal and $-K_{0}$ is an effective divisor,

$$
\begin{aligned}
& \operatorname{an}_{f}\left(\left\lfloor\frac{1}{n} Z-K_{0}\right\rfloor\right) \geq \operatorname{an}_{f}\left(\left\lfloor\frac{1}{n} Z\right\rfloor\right)=Z_{f}, \\
& \operatorname{an}_{f}\left(\left\lfloor Z-K_{0}\right\rfloor\right)=\operatorname{an}_{f}(Z)=Z .
\end{aligned}
$$

Therefore, denoting the ideal $I=H^{0}\left(Y, \mathcal{O}_{Y}(-Z)\right) \subset A$, we have $\mathcal{J}(I) \nsubseteq$ $\mathcal{J}\left(I^{\frac{1}{n}}\right)^{n}$. Thus the strong subadditivity theorem does not hold on $A$.

Remark 2.6. We believe that the strong subadditivity theorem also holds for any two-dimensional regular local ring which is not necessarily essentially of finite type over a field of characteristic zero, but we cannot prove this by an argument on anti-nef cycles such as the proof of Theorem 2.2.

\section{Higher Dimensional CASE}

In the higher dimensional case, we have several counterexamples to Theorem 2.2

Example 3.1. Let $A=\mathbb{C}[[X, Y, Z, W]] /\left(X^{2}+Y^{4}+Z^{4}+W^{5}\right)$ and $\mathfrak{m}=(x, y, z, w)$, where $x, y, z, w$ are the images of $X, Y, Z, W$ in $A$, respectively. Then $A$ is a Gorenstein log terminal singularity, but not a terminal singularity. Therefore, $\mathcal{J}(\mathfrak{m})=\mathfrak{m}$. If $\mathcal{J}\left(\mathfrak{m}^{k+l}\right) \subseteq \mathcal{J}\left(\mathfrak{m}^{k}\right) \mathcal{J}\left(\mathfrak{m}^{l}\right)$ holds for all integers $k, l>0$, then $\mathcal{J}\left(\mathfrak{m}^{n}\right)=$ $\mathfrak{m}^{n}$ for any integer $n>0$, in particular, $\mathfrak{m}^{2}$ should be integrally closed. However, $x \in \overline{\mathfrak{m}^{2}} \backslash \mathfrak{m}^{2}$, because $x^{2} \in \mathfrak{m}^{4}$. This is a contradiction, hence the subadditivity theorem fails on $A$.

Now we investigate the subadditivity theorem for monomial ideals. We expected that the subadditivity theorem for monomial ideals might hold on every toric ring, but unfortunately we found a counterexample on a three-dimensional toric ring.

Example 3.2. Let $M=\left\{(x, y, z) \in \mathbb{Z}^{3} \mid 35 x+28 y+20 z \equiv 0 \bmod 41\right\}$ be a lattice, $\sigma^{\vee}=\left(\mathbb{Z}_{\geq 0}\right)^{\oplus 3} \subset M \otimes_{\mathbb{Z}} \mathbb{R}$ a cone and $A=k\left[M \cap \sigma^{\vee}\right] \subset k[x, y, z]$ the cyclic quotient singularity of type $1 / 41(35,28,20)$. We consider the monomial ideal

$$
I=\left(x^{410}, y^{410}, z^{410}, x^{8} y z, x^{4} y^{6} z, x^{4} y z^{8}\right) \subset A .
$$

Then we will prove that $\mathcal{J}\left(I^{2}\right) \nsubseteq \subseteq \mathcal{J}(I)^{2}$. 
First we will show that $x^{10} y^{3} z^{7} \in \mathcal{J}\left(I^{2}\right)$. Let $P(I)$ be the Newton polygon of $I$, that is, the convex hull of

$$
\{(410,0,0),(0,410,0),(0,0,410),(8,1,1),(4,6,1),(4,1,8)\}
$$

in $M \otimes_{\mathbb{Z}} \mathbb{R}=\mathbb{R}^{3}$. Then note that for every positive integer $n$, by [HY, Theorem 4.8] and [How, $x^{a} y^{b} z^{c} \in \mathcal{J}\left(I^{n}\right)$ if and only if the point $(a+1, b+1, c+1)$ is contained in the interior $\operatorname{Int}(n P(I))$ of $n P(I)$. Since $(10+1,3+1,7+1)=\frac{245}{328}(8,1,1)+$ $\frac{131}{328}(4,6,1)+\frac{281}{328}(4,1,8)$ and $\frac{245}{328}+\frac{131}{328}+\frac{281}{328}>2$, by the above characterization of multiplier ideals associated to monomial ideals, $x^{10} y^{3} z^{7}$ is contained in $\mathcal{J}\left(I^{2}\right)$.

Next we will show that $x^{10} y^{3} z^{7}$ is not contained in $\mathcal{J}(I)^{2}$. If $x^{10} y^{3} z^{7} \in \mathcal{J}(I)^{2}$, then there exist lattice points $(p, q, r),(s, t, u) \in M$ such that $(p, q, r)+(s, t, u)=$ $(10,3,7)$ and $(p+1, q+1, r+1),(s+1, t+1, u+1) \in \operatorname{Int}(P(I))$. Since the three points $(8,1,1),(4,6,1),(4,1,8)$ lie on the plane $35 x+28 y+20 z=328$, by the condition that $(p+1, q+1, r+1),(s+1, t+1, u+1) \in \operatorname{Int}(P(I))$, the lattice points $(p, q, r)$ and $(s, t, u)$ must satisfy that $35 p+28 q+20 r>328-(35+28+20)=245$ and $35 s+28 t+20 u>245$. Moreover, by the assumption $(p, q, r)+(s, t, u)=(10,3,7)$, we know that $(p, q, r)$ and $(s, t, u)$ are obliged to be $(8,1,1)$ and $(2,2,6)$. However, $(2+1,2+1,6+1)$ is not contained in $\operatorname{Int}(P(I))$, because $(2+1,2+1,6+1)=$ $-\frac{83}{328}(8,1,1)+\frac{131}{328}(4,6,1)+\frac{281}{328}(4,1,8)$. This is a contradiction. Thus $x^{10} y^{3} z^{7} \notin$ $\mathcal{J}(I)^{2}$.

Question 3.3. Let $A$ be a Gorenstein toric ring and $\mathfrak{a}, \mathfrak{b}$ be monomial ideals of $A$. Then

$$
\mathcal{J}(\mathfrak{a} \mathfrak{b}) \subseteq \mathcal{J}(\mathfrak{a}) \mathcal{J}(\mathfrak{b}) ?
$$

\section{REFERENCES}

[Ar] M. Artin, On isolated rational singularities of surfaces, Amer. J. Math. 88 (1966), 129-136. MR 33:7340

[DEL] J.-P. Demailly, L. Ein and R. Lazarsfeld, A subadditivity property of multiplier ideals, Michigan. Math. J. 48 (2000), 137-156. MR 2002a:14016

[DV] P. Du Val, On isolated singularities of surfaces which do not affect the conditions of adjunction, Proc. Cambridge Phil. Soc. 30 (1934), 453-459.

[ELS] L. Ein, R. Lazarsfeld, and K. Smith, Uniform bounds and symbolic powers on smooth varieties, Invent. Math. 144 (2001), 241-252. MR 2002b:13001

[Fu] T. Fujita, Approximating Zariski decomposition of big line bundles, Kodai Math. J. 17 (1994), 1-3. MR 95c:14053

[Gi] J. Giraud, Improvement of Grauert-Riemenschneider's Theorem for a normal surface, Ann. Inst. Fourier, Grenoble 32 (1982), 13-23. MR 84f:14025

[HY] N. Hara and K. Yoshida, A generalization of tight closure and multiplier ideals, Trans. Amer. Math. Soc. 355 (2003), no. 8, 3143-3174.

[Hi] H. Hironaka, Resolution of singularities of an algebraic variety over a field of characteristic zero. I, II, Ann. of Math. (2) $\mathbf{7 9}$ (1964), 109-203; ibid. (2) 79 (1964), 205-326. MR 33:7333

[How] J. A. Howald, Multiplier ideals of monomial ideals, Trans. Amer. Math. Soc. 353 (2001), 2665-2671 MR 2002b:14061

[La] R. Lazarsfeld, Positivity in Algebraic Geometry, in preparation.

[Li1] J. Lipman, Rational singularities with applications to algebraic surfaces and unique factorization, Inst. Hautes Études Sci. Publ. Math. 36 (1969), 195-279. MR 43:1986

[Li2] Desingularization of 2-dimensional schemes, Ann. Math. 107 (1978), 151-207. MR 58:10924 
[Li3] , Adjoints of ideals in regular local rings, Math. Res. Lett. 1 (1994), no. 6, 739-755. MR 95k:13028

[Li4] - Proximity inequalities for complete ideals in two-dimensional regular local rings, Commutative algebra: syzygies, multiplicities, and birational algebra (South Hadley, MA, 1992), 293-306, Contemp. Math., 159, Amer. Math. Soc., Providence, RI, 1994. MR 95j:13018

Graduate School of Mathematical Sciences, University of Tokyo, 3-8-1, Komaba, Meguro, TOKYo 153-8914, JAPAN

E-mail address: stakagi@ms.u-tokyo.ac.jp

Department of Mathematics, College of Humanities and Sciences, Nihon University, Setagaya-KU, TOKYO 156-0045, Japan

E-mail address: watanabe@math.chs.nihon-u.ac.jp 